\section{Role of Inertia in Hydrodynamic Lubrication}

$\mathrm{ON}_{\mathrm{N}}$ the basis of the hydrodynamic theory of lubrication presented by Prof. Osborne Reynolds in $1886^{1}$, plane parallel surfaces in relative motion are incapable of developing a load-supporting oil film. This in the direction of motion. Recently, Mr. A. Fogg of the National in the direction of motion. Recently, Mr. A. Fogg of the Nationa Physical Laboratory reported ${ }^{2}$ load capacities for parallel thrust surfaces, operating at high speeds, that were comparable to those obtained with conventional Michell or Kingsbury type thrust bearings. Similar results have also been noted by other researchers ${ }^{3}$.

Mr. Fogg explains these anomalous observations on the basis of a temperature gradient along the oil film in the direction of relative motion. He likens the expansion of the lubricant accompanying this temperature gradient to an equivalent hydrodynamic oil wedge. This
theory is shown to be in qualitative agreement with the observed theory is shown to be in qualitative agreen
data by a process of reasoning by analogy.

data by a process of reasoning by analogy. oil film by lubricant expansion has been made. As Mr. Fogg predicted, oil film by lubricant expansion has been made. As Mr. Fogg predicted, the expansion of the lubricant as it passes through the bearing is
significant, particularly in the case of parallel surfaces. However, the significant, particularly in the case of parallel surfaces. However, the Fogg's observation that at high rotative speeds a pair of parallei plates will carry very nearly the same load as a comparable tilting pad bearing. The analysis rather shows the load capacity of paralle

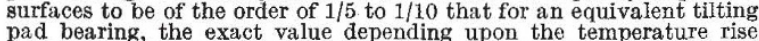
pad bearing, the exact value depending upon the temperature rise and coefficient of expansion obtaining. Although Mr. Fogg's theory are generally designed to support a unit load of approximately $50 \mathrm{lb}$. per sq. in. while tilting pad bearings are designed to carry a load of the order of $500 \mathrm{lb}$. per sq. in., it does not explain the excellent performance of parallel surfaces at high rotative speeds. As Mr. Fogg points out in his paper ${ }^{2}$, the influence of the coefficient of expansion of the lubricant should be included in the analysis of slider and journal bearings which operate with a large rise in temperature.

The unexpected load capacity of parallel plane surfaces can be qualitatively explained on the basis of the inertia-induced pressure developed in an oil film at high rotative speeds. Here the density of the lubricant is the physical property of interest. The inertia of the lubricant has been considered negligible in nearly all hydrodynamic lubrication investigations since the time of Osborne Reynolds. The relatively good agreement between theory and experiment for ordinary slider and journal bearings operating at slow or moderate speeds has amply justified the exclusion of inertia terms. The late Dr. A. Kingsbury ${ }^{4}$ has stressed the need for an investigation of the influence of inertia upon the hydrodynamic characteristics of a bearing. However, a consideration of the magnitude of the centripetal component flm was of negligible importance even for bearings operating at high rotative speeds.

In addition to the centripetal component of acceleration, several other components act upon a particle of oil confined between two parallel rotating disks. These include linear, angular and Coriolis acceleration components. An examination of the hydrodynamic equations including inertia terms qualitatively accounts for the a bearing carries an appreciable load only at relatively high speeds is a bearing carries an appreciable load only at relativel

in agreement with the inertia theory of lubrication. A bearing specially designed to take full advantage of the inertia
effect at all speeds has been built and tested. This device gives appreciably different curves when $\left(\frac{Z N}{P}\right)$ is plotted against the coefficient of friction at several low speeds, where $Z$ is the absolute viscosity of the lubricant in centipoise, $N$ is the speed in r.p.m., and $P$ is the unit load on the projected area of the bearing. Such a multiplicity of friction curves is contrary to ordinary hydrodynamic theory but is in agreement with the inertia theory of lubrication. It is to be expected that careful friction tests of plane parallel disks, thrust bearings of fixed inclination and Michell- or Kingsbury-type bearings will indicate a similar, although smaller, dependence upon speed at high rotative
speeds.

A full MiLTON C. SHAW

Department of Mechanical Engineering,
Massachusetts Institute of Technology, Cambridge, Mass.

Cleveland, Ohio.

Charles D. Strang, Jr. June 20 .

${ }^{1}$ Reynolds, O., Phil. Trans. Roy. Soc., 177, 157 (1886). \& Fogg, A., Engineering, 159, 138 (1945). ' Newbigin, H. T., Proc. Inst. Civil Eng., 196, 223 (1914). Kingsbury, A., Trans. Amer. Soc. Mech. Eng., 50, 6 (1928).
Kingsbury, A., Trans. Amer. Soc. Mech. Eng., 53, 59 (1931).

THE letter from Messrs. Shaw and Strang states that the behaviour of lubricated parallel surfaces, which I reported in a paper to the of lubricated parallel surfaces, which I reported in a paper to the
Institution of Mechanical Engineers in January 1945 , is mainly due Institution of Mechanical Engineers in January 1945, is mainly due to the inertia and not, as I suggested, to thermal expansion of the sion must be included in the analysis of film lubricated bearings, sion must be included in the analysis of film lubricated bearings, they suggest that its effect is only of the order of $1 / 5$ to $1 / 10$ of a this comparison has been made. Has it, as seems probable, been made on the basis of a given mean film thickness with corresponding made on the basis of a given mean film thickness with corresponding relative velocity and oil viscosity ? If so, I suggest that this is not the correct basis for comparison, since the parallel surface bearing will will a fixed taper or tilting pad bearing, for a given degree of surface finish. The flxed-taper surface will obviously fail by contact at the trailing edge when the mean film thickness is relatively large, while experiment shows that the tilting pad bearing, also, fails in the same way. It has, in fact, been shown experimentally that, if taper bearings are cautiously failed a number of times so that the bearing metal is wiped away from the trailing edge without deterioration of the surface condition and a parallel portion established along the surface, the load capacity is thereby increased.

Since my original paper on this subject, further work has shown that the parallel surface thrust bearing functions similarly at low speeds, and, in a number of cases examined, the coefficient of friction $-Z N / P$ curve is substantially the same for the speed range 1,000 to 20,000 r.p.m. In a few cases, with bearing pads of longer arc length, there is some indication that high speed gives a small reduction in friction at constant values of $Z N / P$ well removed from the limiting values. Thus, using the argument of Shaw and Strang that the inertia theory should give a multiplicity of friction $-Z N / P$ curves, and with which I agree, these later experiments indicate that inertia has, at most, a second order effect.

During my earlier. work, which covered the speed-range $10,000-$ 20,000 r.p.m., the possibility of inertia effects accounting for the behaviour was considered and rejected because there was no multiplicity of coefficient of friction $-Z N / P$ curves. It seemed, at first, the obvious explanation, and it was only after much searching that the thermal expansion theory was put forward. Qualitatively, it seemed the only explanation which agreed with the experimental results, and the letter from Shaw and Strang does not convince me otherwise. I shall, however, look forward with great interest to seeing otherwise. I shall, however, look forward with great
their promised full report on their investigations.

Motor Industry Research Association,
Great West Road,

A. FOGG Great West Road,
Brentford, Middlesex. Aug. 24.

\section{Spectroscopic Arguments for Isomeric Structures in a-Chloro-Acids}

IN a recent paper, Renard ${ }^{1}$ has applied a method by which the qualitative analysis of the hydrolysates of proteins seems easily realized. The first step of the process consists in transforming the $a$-mono-amino mono-carboxylic acids resulting from hydrolysis into $a$-chloro-acids; the latter having been separated into different groups by fractional distillation, Raman analysis is used to identify the compounds. The fundamental assumption made by Renard is that
the spectrum of the mixture results from the superposition of the spectra of the constituent acids.

It is perhaps worth while pointing out that the Raman spectra published by Renard have certain characteristic features which indicate that this assumption is unjustifiable. Thus we observe that in each spectrum of the mono-chloro acids (propionic, valeric, isovaleric, caproic, isocaproic, $\beta$-methyl valeric) two large, diffuse bands at about tion. By a nalogy with the behaviour of is presumably due to associathe latter should be correlated with the $\mathrm{C}=0$ frequeney in the monomeric molecule and the former with the same frequency in the dimer. The intensities are difficult to evaluate on the enlargements, but
$1,660 \mathrm{~cm} .^{-1}$ is clearly the most intense line, which indicates considerable polymerization.

It should also be noted that a band situated at about $1,450 \mathrm{~cm} \mathrm{~m}^{-1}$ is a double one. In some cases, we have been able to evaluate the distance between the components at about $20 \mathrm{~cm}^{-1}$. This could possibly be due to the splitting of the simple $\mathrm{C}-\mathrm{O}$ frequency in the
dimer. Many other lines seem to be double, but their exact interpretation would be difficult with the data available at present.

Furthermore, when acids $A$ and $B$ are mixed, the existence of the following equilibria should be considered, namely :

$$
\begin{aligned}
2 A & \rightleftarrows A_{2} \\
2 B & \rightleftarrows B_{2} \\
A+B & \rightleftharpoons A B .
\end{aligned}
$$

$A$ and $B$ being very similar, it seems that $A B$ must play an important part and give rise to new Raman lines the frequencies of which lie between those of $A_{2}$ and $B_{2}$. Thus, Renard's assumption on the additivity of the spectra seems premature, and for this reason alone no qualitative analysis can be undertaken before this point has been cleared up. May we remark that this is only one of the numerous points which, in our opinion, are open to criticism in Renard's paper. Among them, we might mention the precision of the measurements, which is illusory,
and the contradiction between the intensity ratios of the spectral
lines, which do not remain constant in the different mixtures.

University of Liège. Sept. 5 .

1 Renard, M., Mem. Soc. Roy. Sci. de Liège, 7 (1946).

Davies, M. M., and Sutherland, G. B. B. M., J. Chem. Phys., 6, 755 (1938). Herman, R. C., and Hofstadter, R., J. Chem. Phys., $\boldsymbol{\eta}$, 460 (1939).

\section{Fisher's "Problem of the Nile"}

THE following problem is of central importance in the theory of statistical estimation: $k$ unkmown parameters are to be estimated from a sample $S$ consisting of $n$ independent observations from the same parent population; how far can the information in $S$, relevant
to the estimation of the $k$ parameters, be conflned to $k$ degrees of to the estimation of the $k$ parameters, be confined to $k$ degrees of
freedom ? Fisher ${ }^{1}$ has shown that a complete solution is possible 